\title{
HPV INFECTIONS OF THE UTERINE CERVIX IN WOMEN WITH RHEUMATIC DISEASES
}

\author{
N. Nikolov' ${ }^{1}$, T. Totev ${ }^{2}$ \\ ${ }^{1}$ Clinic of Rheumatology, Dr. Georgi Stranski, University Hospital - Pleven \\ ²Department of Obstetrics and Gynecology, Nadezhda Women's Health Hospital - Sofia
}

\begin{abstract}
The relation between systemic autoimmune diseases that affect women in their reproductive age and the neoplasms of the lower genital tract caused by high-risk HPV strains are studied in many investigations. The studies have been focused mainly on patients with systemic lupus erythematosus exposed to glucocorticoids and immunosuppressive therapy. A higher risk of HPV infection persistence and premalignant and malignant cervical lesions formation is found among them. There are similar, though scantier data about other systemic rheumatic diseases - rheumatoid arthritis, Sjögren's syndrome, and systemic sclerosis. Presumably, this fact is determined by specific features of autoimmune inflammation and the impact of immunosuppressive treatment. It contributes to human papilloviruses persistence and their integration into cervical cells. HPV vaccines use in these patients have proved to be safe and effective, and is an object of special interest.
\end{abstract}

Key words: autoimmune rheumatic disease, systemic lupus erythematosus, HPV infection, uterine cervix cancer

\section{INTRODUCTION}

The connection between systemic autoimmune diseases and premalignant and malignant lesions of the lower genital tract due to infections associated with oncogenic human papillomaviruses (HPV) has been investigated in numerous studies. Systemic inflammatory rheumatic conditions affect women, mainly those of fertile age. Most studies in this field are related to systemic lupus erythematosus (SLE). However, patients with rheumatoid arthritis (RA), Sjögren's syndrome (SS), and systemic sclerosis (SSc) are also studied [1, 2].

There are two primary groups of factors that increase the oncogenic risk in patients with autoimmune diseases. The first group is associated with a chronic inflammatory process, while the second is an iatrogenic result from immunosuppressant therapy.

The human papillomavirus (HPV) infection is the most widely spread sexually transmitted infection. It is the primary etiological factor for precancerous and cancerous conditions of the lower genital tract in women. Cervix cancer ranks first among these conditions, followed by carcinoma of the vagina, the vulva, and the anus. There are 40 types of HPV known to cause anogenital lesions, and 15 of them have been recognized as highly oncogenic. In most cases, these infections are transitory and heal spontaneously in $90 \%$ of the cases, usually for one to three years [3]. Persisting highly oncogenic HPV strains contribute to integrating the virus genome into the cervical cells and the initia- tion of a neoplastic process. Immunosuppression and inflammation are among the essential factors for the onset and development of this process, and the risk of persistence and clinical manifestation of HPV infection is much higher in immunocompromised patients than in the general population [4]. An increased risk of premalignant and malignant cervical lesions has been established in patients with systemic lupus erythematosus. There are similar, though scarce, data about the other autoimmune diseases mentioned above $[5,6]$.

\section{Cervical intraepithelial neOplasia AND INVASIVE CERVICAL CANCER}

Carcinogenesis in the uterine cervix occurs as a series of low-grade and high-grade dysplasias, followed by $\mathrm{Ca}$ in situ and invasive cancer. The dysplasias are caused mainly by highly oncogenic strains of the HPV (15 types), and $70 \%$ of the cases are related to types 16 and 18 . The low-grade squamous intraepithelial lesions (LSIL) are present with mild dysplasia that involves the outer one-third of the epithelial layer. The high-grade squamous intraepithelial lesions (HSIL) are moderate and severe dysplasias that affect the deeper epithelial layers. Spontaneous regression of LSIL cases is seen in approximately $70 \%$ of cases, and for HSIL, it ranges from $30 \%$ to $70 \%$ in young women $[7,8]$. In a small number of cases, progress to invasive cancer occurs, and, apart from the type of HPV, many oth- 
er factors play a role. These include age, tobacco smoking, diseases implying weakened immunity, accompanying genital infections, prolonged intake of oral contraceptives, and immunosuppressants.

\section{RISK OF HPV INFECTION AND DEVELOPMENT OF PREMALIGNANT AND MALIGNANT DISEASES OF THE UTERINE CERVIX IN WOMEN WITH RHEUMATIC DISEASES}

HPV invasion is controlled through the systemic and local immune response. It is thought that these viruses do not usually enter the blood circulation, and there is no significant immune response [9]. Antibodies can be detected 3 to 9 months after the infection, and their presence indicates previous exposure. However, these antibodies do not protect against a new infection. Therefore, the only reliable diagnostic method is to detect HPV in infected cervical cells using a PCR test.

Two major factors are assumed to increase the oncogenic risk in patients with rheumatic autoimmune diseases. The activation of autoreactive $T$ and $B$ lymphocytes with the production of proinflammatory cytokines for a prolonged period may trigger carcinogenesis [10]. The role of chronic inflammation as an oncogenic factor was described as early as the 19th century by Rudolf Virchow. Immunosuppression in patients, whether genetic or related to infection (e.g., HIV), or iatrogenic, puts them at high risk for persisting HPV infection. Therapy with glucocorticoids and immunosuppressants reduced the immunity against tumor development. In this aspect, women with autoimmune rheumatic diseases are predisposed to persisting HPV infection and the development of precancerosis and cervical cancer [11].

Such assumptions have been confirmed in many studies. More than two types of HPV and a prevalent carriage of highly oncogenic types were seen in SLE patients compared to the healthy population [12].

There are significantly more frequent abnormal cytologic findings after PAP smear tests in women with SLE than healthy ones [11, 13]. Similar data have been reported for patients with RA [14]. There were no significant differences between the study group and the controls in patients with Sjögren's syndrome.

Several studies have confirmed an increased risk of precancerosis (SIL), associated with HPV infection in women with SLE $[6,12]$. Similar data have been reported about the higher prevalence of premalignant lesions in patients with other rheumatic diseases - RA and SS compared to healthy populations [15, 16].

A large cohort study $(n=4976)$ found that SLE is a risk factor for the development of SIL but not of invasive cancer [12]. A cohort study of an identical design in the same country (Sweden) has established an increased risk (40-50\%) for LSIL and HSIL in RA patients, and no association with the development of invasive cancer of the uterine cervix was found [17]. Such results are probably due to well-organized mass screening and adequate treatment of precancerosis.

Of interest is the combination of systemic rheumatic disease and other risk factors for precancerous and cancerous lesions of the uterine cervix. The presence of several risk factors significantly increases the risk in such patients. A study on RA patients has found that in $29 \%$ of them, the results of a PAP smear test were abnormal, and other registered risk factors included three or more sex partners, history of another sexually transmitted infection, and previous oral contraception [18].

A multivariate analysis performed by Klumb et al. has shown that SLE patients present with a seven-fold higher presence of LSIL and an eleven-fold higher presence of HSIL than controls. These findings identify an independent factor for abnormal smear test results in cases of three or more sexual partners $(O R=2.44)$ and those with SLE $(O R=$ 7.08) [11].

Skare et al. have reported an increased prevalence of cervical cancer in women with SLE, as compared with healthy ones ( $p<0.0001$, OR 10.4), as well as an association with the duration of the disease $(p=0.006)$ [19].

\section{ROLE OF IMMUNOSUPPRESSIVE THERAPY}

Several studies have provided evidence that exposure to prednisone and long-term immunosuppressive therapy (cyclophosphamide) increases the risk for the onset and persistence of precancerous conditions of the uterine cervix [11, 13, 20]. A cohort study on patients with various autoimmune diseases established an increased incidence of cervical cancer following treatment with azathioprine and an association with a higher cumulative dose $(H R=2.2)$ and duration of therapy ( $\geq 5$ years, HR $=3.3$ ) [20]. No increased risk of onset and persistence of HPV genital infections were found in RA patients undergoing anti-TNF treatment [14]. 


\section{APPLICATION OF HPV VACCINES IN PATIENTS WITH RHEUMATIC DISEASES}

Three vaccines based on virus-like particles that include L1 HPV protein are currently in use. The most common vaccines include a bivalent one (for HPV types 16 and 18) and a quadrivalent one (for HPV types $6,11,16,18)$. There is also 9-valent one (for HPV types 6, 11, 16, 18, 31, 33, 45, 52 и 58). The recommended age for vaccination is 11-12 years, and application between ages 9 and 26 is also acceptable. Covering at least $75 \%$ of the relevant population contingent is necessary to make the vaccination effective. The bivalent and the quadrivalent vaccines help maintain high anti-HPV antibody levels for at least 10 years [21].

In general, it is assumed that there are no contraindications for vaccination in immunocompromised patients, except in cases of live vaccines. According to data provided by CDC, however, $60 \%$ of girls receive the first dose, and only $40 \%$ get the recommended series of three doses [22]. Data from a previous period show that only $21 \%$ of patients with rheumatic diseases have started immunization against HPV infection. As a rule, there is an adequate immune response in women with autoimmune rheumatic diseases. The antibody level in patients with SLE is similar to that in the healthy population after 6 to 12 months and does not decrease in patients on steroids, AZA, ciclosporin, tacrolimus, and HCQ 18 months after vaccination. Only those treated with mycophenolate have immunity as low as $33 \%$ within that period [23].

There remains the question of vital importance whether immunization with vaccines against HPV triggers autoimmune diseases. There are very few cases of onset of such diseases after immunization reported in the literature [24]. A cohort study on patients with autoimmune diseases who received quadrivalent HPV vaccine did not register an increased risk for onset of another autoimmune disease [25]. A systematic review of five studies on women of fertile age with autoimmune diseases found no exacerbation of the conditions was found after vaccination [26].

\section{Cervical cancer screening FOR WOMEN WITH AUTOIMMUNE RHEUMATIC DISEASES}

There exists an opinion based on studies that precancerous and cancerous growths in the uterine cervix are not diagnosed on time in women with systemic rheumatic diseases. There is a scarcity of officially designed guidelines focusing on mass cervical cancer screening for patients in this group and different from approaches oriented to low-risk populations. Based on the principle that they are exposed to long-term immunosuppressive therapy, it is suggested that rules like those applying to HIV-positive women should be observed. These rules would imply a Pap test every year for three years. If test results are within a normal range, then the period between tests is extended to three years, and HPV investigation (cervical fluid PCR test) at the age of $\geq 30$. Screening is to be started at the 21 st year or earlier in case of earlier sex life [21].

\section{Conclusion}

Women with autoimmune rheumatic diseases receiving immunosuppressive therapy are at a higher risk for persisting HPV infection of the lower genital tract. They are more often prone to the onset of premalignant lesions that may lead to invasive cancer development. There are two basic approaches to reduce the incidence and death rate related to uterine cervical cancer. The first one is mass and timely HPV vaccination since the currently used vaccines have proved to be effective and safe in women with rheumatic diseases. The other is adequate cytological screening and treatment of diagnosed precancerous lesions.

\section{Библиография / References}

1. Dalstein V, Riethmuller D, Pretet JL et al. Persistence and load of high-risk HPV are predictors for development of highgrade cervical lesions: a longitudinal French cohort study. Int J Cancer 2003;106:396-403.

2. Marrow J, Nelson L, Watts R et al. Autoimmune Rheumatic Disease. Oxford University Press: Oxford. 1999.

3. Ho GY, Bierman R, Beardsley L et al. Natural history of cervicovaginal papillomavirus infection in young women. N Engl J Med 1998;338:4238.

4. Lee YH, Choe JY, Park SH et al. Prevalence of human papilloma virus infections and cervical cytological abnormalities among Korean women with systemic lupus erythematosus. J Korean Med Sci. 2010;25(10):1431-1437.

5. Santana IU, Gomes Ado N, Lyrio LD et al. Systemic lupus erythematosus, human papillomavirus infection, cervical premalignant and malignant lesions: a systematic review. Clin Rheumatol. 2011;30(5):665-672.

6. Zard E, Arnaud L, Mathian A et al. Increased risk of high grade cervical squamous intraepithelial lesions in systemic lupus erythematosus: A meta-analysis of the literature. Autoimmun Rev. 2014;13(7):730-735.

7. Wheeler CM. Natural history of human papillomavirus infections, cytologic and histologic abnormalities, and cancer. Obstet Gynecol Clin North Am 2008;35:51936.

8. Moscicki AB, Shiboski S, Hills NK et al. Regression of lowgrade squamous intra-epithelial lesions in young women. Lancet 2004;364:167883. 
9. Stanley MA. Epithelial cell responses to infection with human papillomavirus. Clin Microbiol Rev 2012;25:21522.

10. Copper GS, Stroehla BC. The epidemiology of autoimmune diseases. Autoimmun Ver. 2003;2:119-125.

11. Klumb EM, Araújo Jr ML, Jesus GR et al. Is higher prevalence of cervical intraepithelial neoplasia in women with lupus due to immunosuppression? J Clin Rheumatol. 2010;16(4):153-157.

12. Wadstrom H, Arkema EV, Sjowall C et al. Cervical neoplasia in systemic lupus erythematosus: a nationwide study. Rheumatology 2016;56:6139.

13. Rojo-Contreras W, Olivas-Flores EM, Gamez-Nava Jl et al. Cervical human papillomavirus infection in Mexican women with systemic lupus erythematosus or rheumatoid arthritis. Lupus. 2012;21(4):365-372.

14. Mercado U. Abnormal cervicovaginal cytology in women with rheumatoid arthritis. Ginecol Obstet Mex. 2010;78(2):94-98.

15. Kim SC, Glynn RJ, Giovannucci E et al. Risk of high-grade cervical dysplasia and cervical cancer in women with systemic inflammatory diseases: a population-based cohort study. Ann Rheum Dis 2015;74:13607.

16. Raposo A, Tani C, Costa J et al. Human papillomavirus infection and cervical lesions in rheumatic diseases: a systematic review. Acta Reumatol Port 2016;41:18490.

17. Wadstrom H, Frisell T, Sparen $P$ et al. Do RA or TNF inhibitors increase the risk of cervical neoplasia or of recurrence of previous neoplasia? A nationwide study from Sweden. Ann Rheum Dis 2016;75:12728.

18. Gillet VG, Solomon DH, Shadick NA et al. Behavioral and clinical factors associated with self-reported abnormal Papanicolaou tests in rheumatoid arthritis. J Womens Health 2014;23:7716.

Постъпил за печат: 30.11.2020 2.

$\triangle$ Адрес за кореспонденция:

Д-р Т. Тотев

e-mail: t.totev@mail.bg
19. Skare TL, da Rocha BV. Breast and cervical cancer in patients with systemic lupus erythematosus. Rev Bras Ginecol Obstet. 2014 Aug;36(8):367-371.

20. Dugué PA, Rebolj M, Hallas J et al. Risk of cervical cancerin women with autoimmune diseases, in relation with their use of immunosuppressants and screening: populationbased cohort study. Int J Cancer. 2015;136(6):E711-719.

21. Kim SC, Feldman S, Moscicki AB. Risk of human papillomavirus infection in women with rheumatic disease: cervical cancer screening and prevention. Rheumatology 2018;57:v26v33.

22. Reagan-Steiner S, Yankey D, Jevaraiah J et al. National, regional, state and selected local area vaccination coverage among adolescents aged 13-17 years United States, 2015. Morb Mortal Wkly Rep 2016;65:8508.

23. Mok CC, Ho LY, Fong LS et al. Immunogenicity and safety of a quadrivalent human papillomavirus vaccine in patients with systemic lupus erythematosus: a case-control study. Ann Rheum Dis 2013;72:65964.

24. Macartney KK, Chiu C, Georgousakis M et al. Safety of human papillomavirus vaccines: a review. Drug Saf 2013;36:393412.

25. Gronlund O, Herweijer E, Sundstrom K et al. Incidence of new-onset autoimmune disease in girls and women with pre-existing autoimmune disease after quadrivalent human papillomavirus vaccination: a cohort study. J Intern Med 2016;280:61826.

26. Pellegrino P, Radice S, Clementi E. Immunogenicity and safety of the human papillomavirus vaccine in patients with autoimmune diseases: a systematic review. Vaccine 2015;33:34449.

Submitted: 30.11 .2020

Correspondence address:

T. Totev, MD

e-mail: t.totev@mail.bg 